\title{
Study on Size-Related Product Quality of Multiscale Central-Punched Cups Fabricated by Compound Forming Directly Using Brass Sheet
}

\section{Dien $\mathrm{Hu}$}

The Hong Kong Polytechnic University https://orcid.org/0000-0001-7165-4050

Jun-Yuan Zheng

The Hong Kong Polytechnic University

M.W. Fu ( $D$ mmmwfu@polyu.edu.hk)

The Hong Kong Polytechnic University

\section{Research Article}

Keywords: Meso/microformed parts, Compound forming, Size effect, Deformation behaviors,

Dimensional accuracy, Surface quality.

Posted Date: January 14th, 2022

DOI: https://doi.org/10.21203/rs.3.rs-1254478/v1

License: (c) (i) This work is licensed under a Creative Commons Attribution 4.0 International License. Read Full License

Version of Record: A version of this preprint was published at The International Journal of Advanced Manufacturing Technology on April 23rd, 2022. See the published version at https://doi.org/10.1007/s00170-022-09222-z. 


\title{
Study on Size-related Product Quality of Multiscale Central-Punched Cups \\ Fabricated by Compound Forming Directly Using Brass Sheet
}

\author{
Dien Hu, Jun-Yuan Zheng, M.W. Fu ${ }^{*}$ \\ *mmmwfu@polyu.edu.hk \\ Department of Mechanical Engineering, The Hong Kong Polytechnic University, \\ Hung Hom, Kowloon, Hong Kong, China
}

\begin{abstract}
Meso/microforming has gained much more attention in the last decades and is widely used as a reliable method to fabricate meso/micro-scaled metallic components. In this research, a compound meso/microforming system which combines deep drawing, punching and blanking operations was developed to fabricate multiscale central-punched cups by using brass sheets. The parts with three scales were produced by using the brass sheets with various thicknesses and grain sizes to investigate geometrical and grain size effects on the deformation behaviors, dimensional accuracy, and material flow behaviors in the forming process. Through physical experiments and finite element simulations, it is revealed that the ultimate deformation load in the drawing-punching stage is smaller than that in the single deep drawing stage under microscale, but the results in the meso-scaled scenarios are opposite. In addition, the thickness variation is increased with grain size, but the variation of the normalized thickness variation does not show an obvious tendency with different size scales. In the bending area, the material flow is tangential to the thickness direction, leading to the formation of thinning area. In addition, the material flow is almost opposite to the punching direction in the punching area, avoiding the expanding deformation of the hole. Thus, the punching operation barely affects the dimensional accuracy including the thickness and hole diameter of the formed parts. Furthermore, the micro-scaled cups with finer grains have a better surface quality. These findings enhance the understanding of size effect in compound meso/microforming with the combined deep drawing and punching operations.
\end{abstract}

Keywords: Meso/microformed parts; Compound forming; Size effect; Deformation 
behaviors; Dimensional accuracy; Surface quality.

\section{Introduction}

Nowadays, meso/micro-scaled components are in great demand and widely applied in a variety of industrial fields [1]. Meso/microforming based on plastic deformation is one of the effective and efficient manufacturing methods to fabricate meso/micro-scaled parts and structures with the advantages of low cost, high efficiency and more environment-friendly [2]. The fabricated multiscale parts are widely used in electrical/electronic, micro-electro-mechanical systems (MEMS), consumer electronics, and bio-medical fields [3]. Due to the existence of size effect with the scaling down of part size, the knowledge and know-how in macromanufacturing cannot be fully applicable to meso/micromanufacturing, which does not facilitate and promote the applications of meso/microforming processes [4].

To address the size effect induced processing deviations, researchers have studied much on the size effect affected process performances and part quality of meso/microforming. Computer-aided simulation can help the evaluation, analysis and comparison of different design solutions and the related outcomes, and further identify the good, better and best solutions [5]. In tandem with this, researchers have developed different theoretical models to predict and analyze the process behaviors and performances related to size effect in different meso/microforming processes. To name a few, Lai et al. [6] established a hybrid model that combined the surface layer model with polycrystal and single-crystal theories to study size effect in microforming process. Peng et al. [7] constructed a constitutive model based on a uniform size dependence to predict the evolution of material behaviors from macroscale to microscale. Wang et al. [8] established the polycrystalline model considering the rough surface to evaluate the surface quality of the parts fabricated by microforming. Zhang et al. [9] established a crystal plasticity based constitutive model to study influences of the size effect factors on the plastic anisotropy and fracture failure of metal foils.

In addition to these methodology-based explorations, the physical experiments 
and finite element (FE) simulations were also widely used to develop the understanding of the size effect dependent micro/mesoforming. Klein et al. [10] observed the stress-strain behaviors of thin metallic foils by micro-tensile experiments and revealed that size effect mainly influences fracture due to the differences of texture. Zhang et al. [11] observed the microtension of polycrystalline copper foils with various thicknesses and grain sizes by both physical experiments and FE simulations, and discovered that grain size, sample scale, and crystallographic orientation significantly affect the mechanical response of the foils in a synthetic way. Chan et al. [12] conducted embossing experiments and tensile tests of microchannels fabricated by pure copper foils and discovered that surface roughness was increased with the grain size of the foils. Furthermore, Jiang et al. [13] conducted microcompression experiments to investigate the effects of temperature and grain size in this process and revealed that when the workpieces are made up of fine grains, the mechanical behaviors of the workpieces are more sensitive to working temperature. Feng et al. [14] conducted the cutting experiments by using three types of sheet metals and revealed that a non-proportional decrease of friction leads to size effect arising. Especially, Chan et al. [15] developed a combined analysis methodology based on artificial neural networks and FE simulations to predict and evaluate the performances of the processes.

In addition, some other microforming processes have also been extensively explored. Among them, Luo et al. [16] conducted micro-hydromechanical deep drawing experiments and FE simulation and found that hydraulic pressure and size effect influence the height and number of wrinkles. Molitnikov et al. [17] studied the deep drawing process considering the blank thickness and discovered that the size effect can be influenced by the ratio of grain size and the blank thickness in this process. Chan et al. [18] further investigated the size effect on the forging of flanged components and developed a systematic design approach for predicting and avoiding folding defects. Then Chan et al. [19] studied the manufacturing of meso-scaled flanged parts and cylindrical bulk. It is revealed that there are fracture and shearing 
surfaces both on the two parts, and the better quality of the fabricated parts can be obtained with the fine-grained materials. Xu et al. [20] conducted micro-blanking tests to study the size effect in this process using brass foil and revealed that ultimate shear strength increases when the thickness of the material decreases and the deformation behaviors of the material are linked to the clearance and grain size. Moreover, Zheng et al. [21-23] designed and developed several microforming systems including compound and progressive microforming to fabricate parts with different features, scales and grain sizes to investigate deformation behaviors, material flow phenomena and the undesirable geometries formed in the fabricated parts. They also revealed the size effect on different multiscale forming processes, which offered a research foundation for future research on compound and progressive microforming.

Some other researchers also investigated the process performance and product quality in deep drawing and punching processes. Raabe et al. [24] investigated the effect of surface roughness on the earing behaviors of low carbon steel in deep drawing process based on the crystal plasticity finite element method (CPFEM) considering material texture. Li et al. [25] conducted micro deep drawing experiments which were realized in two steps. It was found that fracture in the thinning area can easily happen, and the forming load of fine-grained material is larger in the process. Luo et al. [26] conducted deep drawing experiments and FE simulations with various blank holder gaps and grain sizes and revealed that the relatively small gap leads to the good quality of the wall without the increase of fracture. Chen et al. [27] conducted deep drawing experiments and obtained the limit drawing rations (LDR) of brass foils with various gran sizes, then adopted two macro-empirical equations for predicting forming load and LDR of the cup. It was found that the LDR is influenced by thickness, grain size, thickness to grain size (T/D) ratios and other factors, but the equations can only predict the deformation of the foils thicker than $150 \mu \mathrm{m}$. Gong et al. [28] developed the diamond-like carbon (DLC) film covered blank holders for the deep drawing process and revealed that DLC films are proven to reduce drawing force, enhance LDR and improve lubricating conditions. Fu et al. [29] developed a 
compound deep drawing microforming system including blanking and deep drawing and verified the feasibility of the system. It was discovered that the forming load increases with the decrease of grain size if there are a sufficient number of grains in the thickness direction in the deep drawing process. Vollertsen and $\mathrm{Hu}$ [30] adopted various punching velocities for experiments and found that velocities lead to the differences of friction coefficients, which cause different process windows. Joo et al. [31] designed a micro punching press for $25 \mathrm{um}$ holes and identified that micropunching has the ability to manufacture holes of good quality.

As mentioned above, much research has been conducted on size effect and the processes of deep drawing and punching. However, it still lacks the study on the size effect in compound meso/microforming processes, which combine deep drawing, punching and blanking in different size scales using the materials with various grain sizes. In this research, a compound forming system including deep drawing, punching and blanking operations with several size scales was designed and developed to fabricate the central-punched cups. The size-related deformation behaviors, dimensional accuracy and the influence of processes were investigated from the aspects of part and grain size scales.

\section{Materials and experiments}

\subsection{Experimental material and mechanical properties}

Brass CuZn25 was used as the testing material in this study since it has good ductility and electrical conductivity and is widely used for making different electronic parts and components. The 20 by $100 \mathrm{~mm}$ brass sheets with thicknesses of $0.2,0.4$ and $0.6 \mathrm{~mm}$ were annealed in an argon-filled furnace under three heat treatment conditions, viz. 500,550 , and $650{ }^{\circ} \mathrm{C}$ for $1 \mathrm{~h}$, to achieve different grain sizes. A solution of $5 \mathrm{~g} \mathrm{FeCl}_{3}, 15 \mathrm{ml} \mathrm{HCl}$ and $85 \mathrm{ml} \mathrm{H}_{2} \mathrm{O}$ was prepared to etch the heat-treated samples for $10-15 \mathrm{~s}$. The grain sizes of the sheets were measured using a metallographic microscope. The microstructures of the brass sheets are shown in Fig. 1. Table 1 gives the grain sizes with different thicknesses of the specimens and their related annealing temperatures measured by the lineal intercept technique following 
the ASTM E112 standard. Obviously, the high annealing temperature results in coarse grains.

Table 1 Annealing temperature and the corresponding grain sizes of the sheets with different thicknesses

\begin{tabular}{|c|c|c|c|c|c|c|c|c|c|}
\hline & \multicolumn{7}{|c|}{ Annealing temperature } \\
\cline { 2 - 9 } & \multicolumn{3}{|c|}{$500^{\circ} \mathrm{C}$} & \multicolumn{3}{c|}{$550^{\circ} \mathrm{C}$} & \multicolumn{3}{c|}{$650^{\circ} \mathrm{C}$} \\
\hline $\begin{array}{c}\text { Thickness } \\
(\mathrm{mm})\end{array}$ & 0.2 & 0.4 & 0.6 & 0.2 & 0.4 & 0.6 & 0.2 & 0.4 & 0.6 \\
\hline $\begin{array}{c}\text { Grain size } \\
(\mu \mathrm{m})\end{array}$ & 37 & 39 & 44 & 60 & 62 & 70 & 121 & 154 & 156 \\
\hline
\end{tabular}
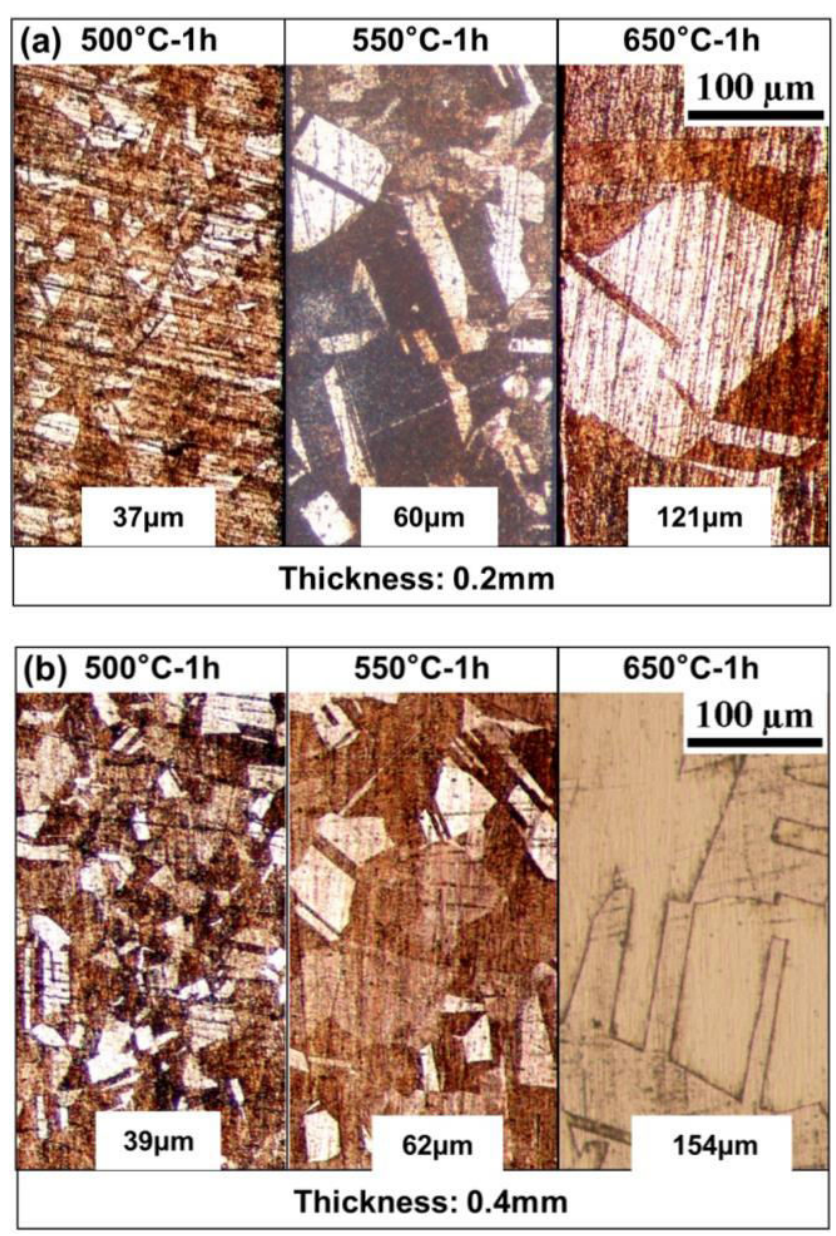


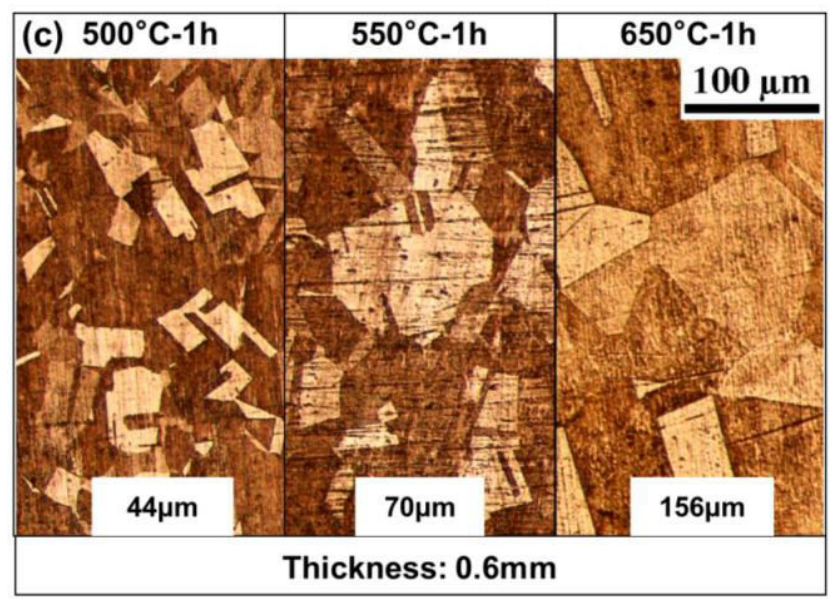

Fig. 1 The initial microstructures of brass sheets under different annealing conditions: the thickness: (a) $0.2 \mathrm{~mm}$, (b) $0.4 \mathrm{~mm}$, and (c) $0.6 \mathrm{~mm}$

Tensile tests were conducted on an MTS machine following ASTM E8/ E8M to get the flow stress and plastic strain curves of the testing materials. Dog-bone sample shown in Fig. 2 (a) was produced for tensile tests. The uniaxial tensile tests were performed three times for each testing material. The flow stress and plastic strain curves are shown in Fig. 2 (b-d). It is found that the flow stress of fine-grained material is larger, following the Hall-Petch relationship [32]. 
(a)

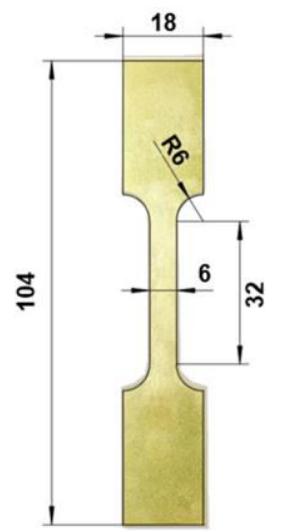

(c)

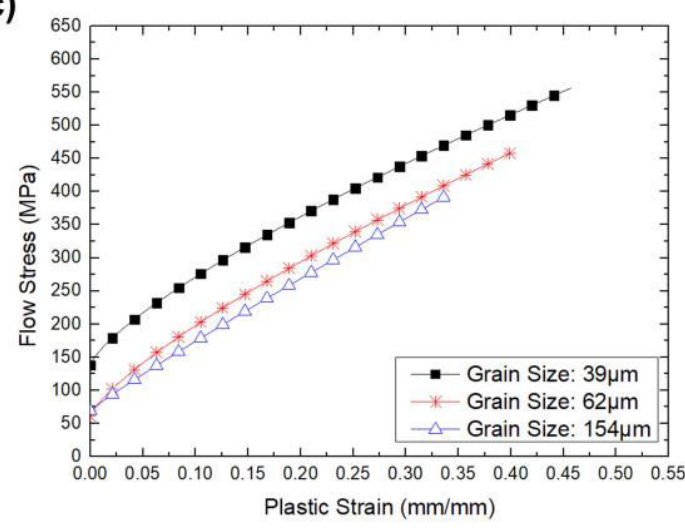

(b)

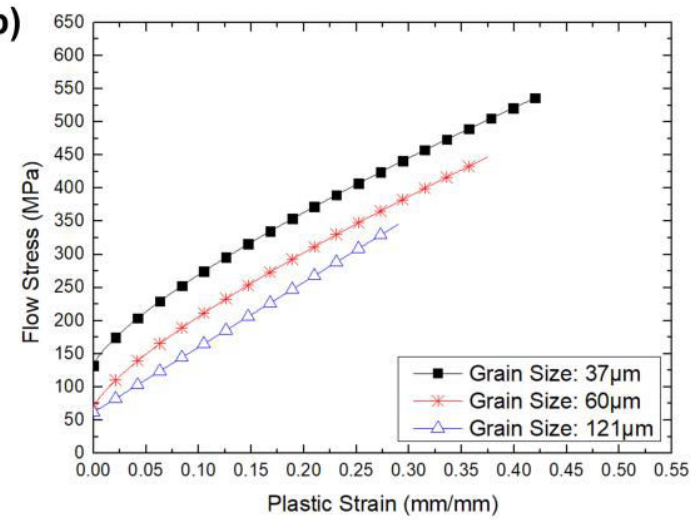

(d)

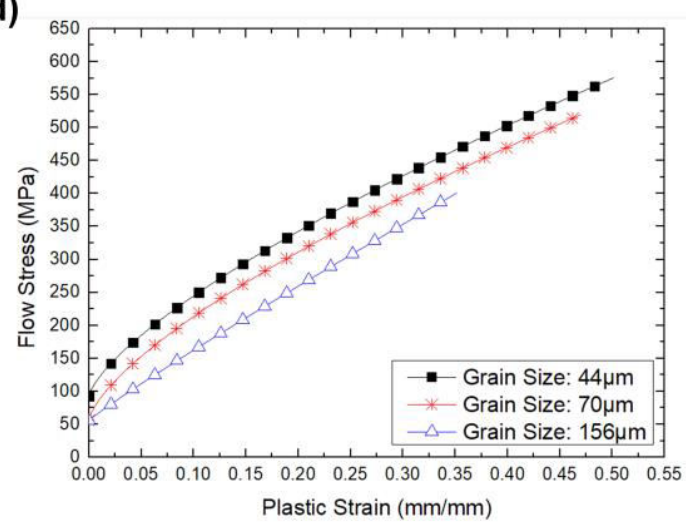

Fig. 2 (a) Illustration of dog-bone samples. Stress-strain curves of the brass CuZn25 with various grain sizes and the thickness of (b) $0.2 \mathrm{~mm}$, (c) $0.4 \mathrm{~mm}$, and (d) $0.6 \mathrm{~mm}$

\subsection{The compound meso/microforming}

A compound forming system combining blanking, deep drawing and punching operations within one stroke was developed, as shown in Fig. 3 (a) and (c). The schematic diagrams of the forming system are given in Fig. 3 (b) and (d). In the beginning, the blank holder is fixed to constraint the brass sheet. The upper punch then moves downward, and a round workpiece is cut from the brass sheet and fell into the bottom die. The deep drawing operation subsequently starts. When the workpiece is deformed to half a cup by deep drawing, the upper punch contacts the workpiece. Meanwhile, the deep drawing and punching are conducted simultaneously. The upper punch continues to move until the final central-punched cup is fabricated. 
(a)

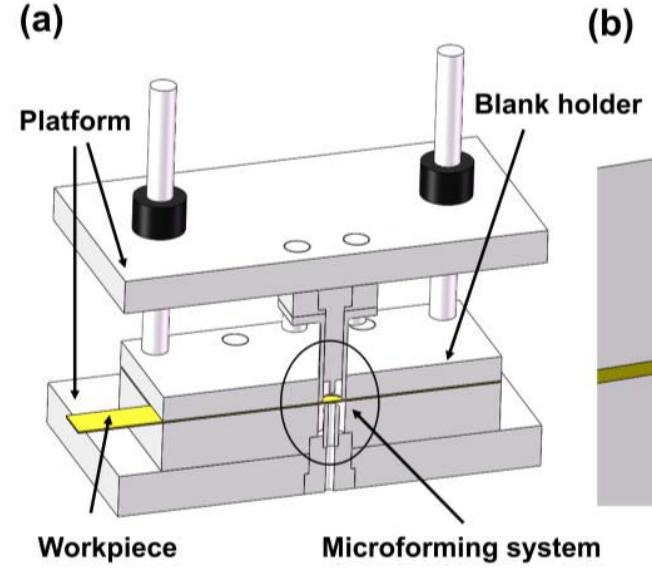

(c)

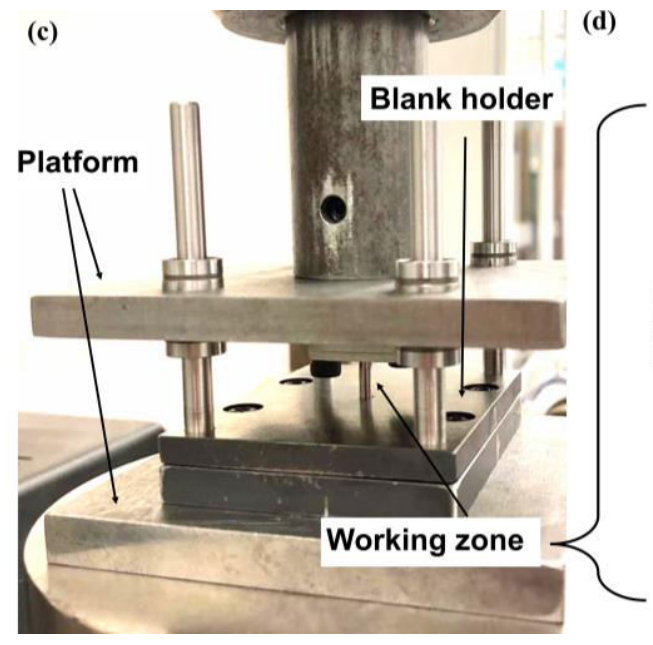

B) Blanking punch\& drawing die

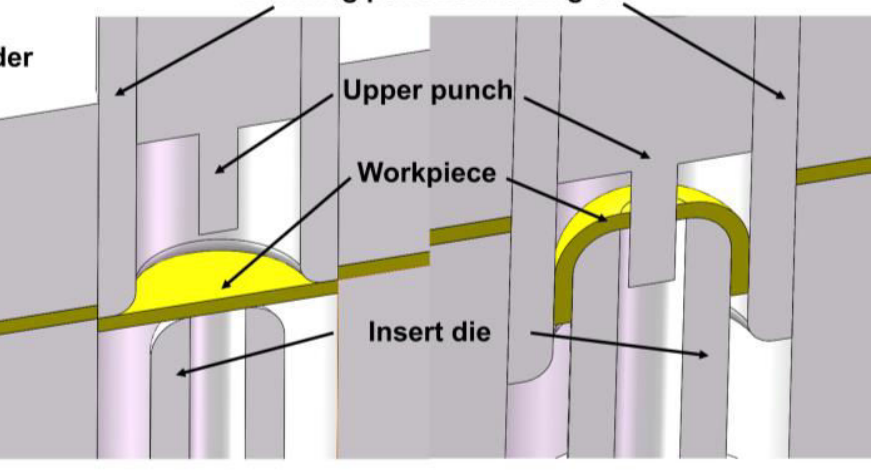

(d)

Fig. 3 (a) Illustration of the forming system. (b) Illustration of the process of the forming system. (c) Physical view of the compound forming system, and (d) the physical objects of each die

The central-punched cups with 3 size scales including microscale and two mesoscales were designed with the scale factor $\alpha$ of $0.5,1$ and 1.5 with the corresponding brass sheet thickness of $0.2,0.4$, and $0.6 \mathrm{~mm}$, respectively. Their specific sizes are shown in Table 2. A forming system was designed for making different scale parts via changing the different working components. The specific dimensions of the dies with different size scales are shown in Table 3.

Table 2 Dimensions of the parts with different size scales (mm) 


\begin{tabular}{|c|c|c|c|}
\hline$\alpha$ & 0.5 & 1 & 1.5 \\
\hline $\mathrm{d} 1$ & 2.2 & 4.4 & 6.6 \\
\hline $\mathrm{d} 2$ & 0.6 & 1.2 & 1.8 \\
\hline $\mathrm{h} 1$ & 0.6 & 1.2 & 1.8 \\
\hline $\mathrm{r} 1$ & 0.6 & 1.2 & 1.8 \\
\hline $\mathrm{t}$ & 0.2 & 0.4 & 0.6 \\
\hline
\end{tabular}

Table 3 Dimensions of the die with different size scales $(\mathrm{mm})$

\begin{tabular}{|c|c|c|c|c|}
\hline$\alpha$ & 0.5 & 1 & 1.5 \\
\hline $\mathrm{D} 1$ & 1.8 & 3.6 & 5.4 \\
\hline $\mathrm{D} 2$ & 0.8 & 1.6 & 2.4 \\
\hline $\mathrm{D} 3$ & 0.6 & 1.2 & 1.8 \\
\hline $\mathrm{D} 4$ & 2.2 & 4.4 & 6.6 \\
\hline $\mathrm{D} 5$ & 3.2 & 6.4 & 9.6 \\
\hline $\mathrm{R} 1$ & 0.5 & 1 & 1.5 \\
\hline $\mathrm{R} 2$ & 0.3 & 0.6 & 0.9 \\
\hline $\mathrm{L} 1$ & 0.2 & 0.4 & 0.6 \\
\hline $\mathrm{L} 2$ & 1 & 2 & 3 \\
\hline $\mathrm{t}$ & 0.2 & 0.4 & 0.6 \\
\hline
\end{tabular}

Note: D1, diameter of insert die; D2, diameter of punch hole; D3, diameter of upper punch; D4, diameter of drawing die; D5, diameter of blanking punch; R1, radius of insert die; R2, radius of drawing die.

Table 4 Punching speed at different size scales

\begin{tabular}{|c|c|}
\hline Scale factor $\alpha$ & Punch Velocity $(\mathrm{mm} / \mathrm{s})$ \\
\hline 0.5 & 0.01 \\
\hline 1 & 0.02 \\
\hline 1.5 & 0.03 \\
\hline
\end{tabular}

The whole process was realized in an MTS testing machine with a $50 \mathrm{kN}$ load capacity. Machine oil was used as lubricant on the interfaces. Slow punching speeds were selected and thus the influence of the strain rate effect can be ignored. The 
punching speeds of different size scales are shown in Table 4. The loading data were obtained by averaging the multiple sets of experimental results. The central-punched cups fabricated by sheets of different thicknesses annealed at $500^{\circ} \mathrm{C}$ are shown in Fig.

4.

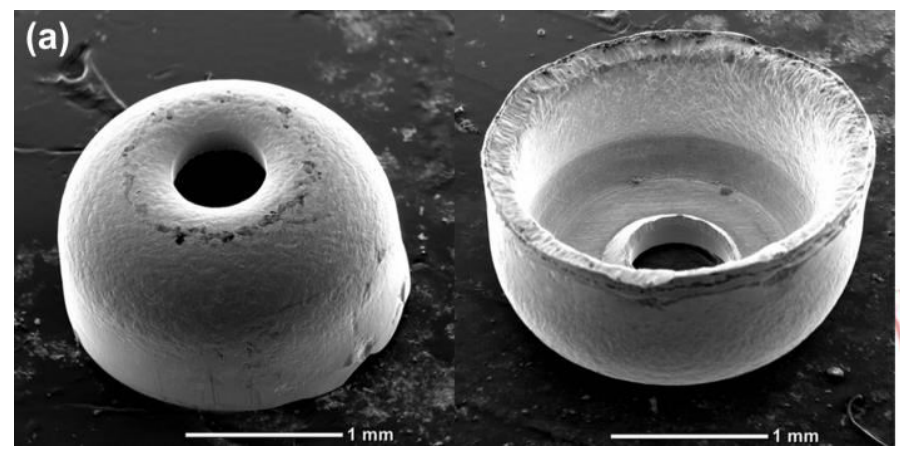

(b)

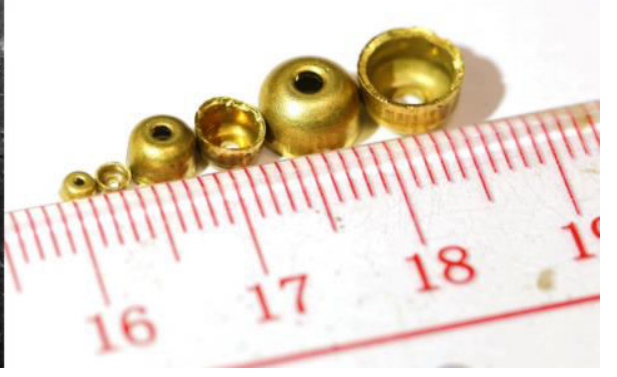

Fig. 4 (a) Part photographed by scanning electron microscope (SEM) with the size scale of 0.5. (b) The central-punched cups with different scales

\section{Results and discussion}

\subsection{Deformation behavior}

The normalized load-stroke curves can well represent the deformation behaviors of materials during the forming process with a better comparison between different size scales. The deformation load and stroke in the experiments were normalized using the following equations [29].

$$
\begin{gathered}
\text { Scaled deformation load }=\frac{\text { punching force }}{\alpha^{2}} \\
\text { Scaled punch stroke }=\frac{\text { punch stroke }}{D 1}
\end{gathered}
$$

The load-stroke curves with different size scales and grain sizes are shown in Fig. 5. The whole process can be divided into three stages, viz. blanking, deep drawing and drawing-punching stages. The deformation load in the first stage has a rising and then falling trend, similar to the trend in the conventional blanking process. A regular circular blank is cut off from the brass sheet in this stage. The second stage is similar 
to other deep drawing processes with a single-peaked shape [26], and the circular blank gradually takes the shape of a cup during this process. In the third stage, the curve tends to rise again due to the beginning of punching.

Fig. 5 (b) shows the deformation behaviors of the cups with various grain sizes and the same thickness. The deformation load is decreased with the increase of grain size. The grain boundary strengthening effect can be employed to explain this phenomenon, in which the grain boundaries serve as an obstacle to the movement of dislocation [33]. Coarse-gained materials have less grain boundary, leading to less restriction to deformation. For fine-grained materials, more grain boundaries induce dislocation stacking, which further results in the strengthening or hardening of materials. Thus, for the materials with the same thickness, the load is smaller for the materials with larger grains. Fig. 5 (c) illustrates the deformation load with the similar grain size and different thicknesses has similar trends. However, there is a deviation between microscale and mesoscale in the third stage. In this stage, the ultimate scaled load of microscale is smaller than its ultimate load in the second stage. In addition, the scaled load of microscale in the third stage is much smaller than that of mesoscale when their strokes are the same. The surface layer model can be used to explain these phenomena. The surface grains are less constrained than the internal grains, and micro-scaled sheets have several to dozen grains in the thickness direction, which leads to less restriction of the deformation [34]. Moreover, during the forming of the cups, the stress states of the two operations are not the same; shear is the main deformation mechanism in punching operations, while tension is the main deformation mode in deep drawing operation. These would result in the differences in deformation load between microscale and mesoscale during the third stage. Furthermore, the small workpiece size leads to uneven lubrication on the surface, which can be explained by open lubrication pocket (OLP) and closed lubrication pocket (CLP) theories [35]. Thus, the differences in the second and third stages between microscale and mesoscales may be due to the complex friction condition, and the punching force in microscale may play a less important role in the deformation 
load. As to the meso-scaled parts, the difference in the scaled forming load is induced by the different percentages of surface grains, which can be explicable by the surface layer model. The $0.6 \mathrm{~mm}$ sheets have more internal grains in the thickness direction, which leads to a higher deformation load. In addition, a numerical analysis of the process was conducted by DEFORM. The coefficient of friction in the simulation is 0.1 , and the punch speeds are the same as those in the actual experiment given in Table 4. Fig. 5 (d) shows the load-stroke curves through FE simulations and experiments with various thicknesses and similar grain sizes. It was revealed that the simulation results follow the same pattern as the experimental findings but with certain deviations. On the one hand, the material is unevenly deformed during the forming process due to its inhomogeneous property. On the other hand, the actual forming process is more complex in terms of friction [25]. These situations were not considered in the simulation.

(a)

Stage I Stage II Stage III

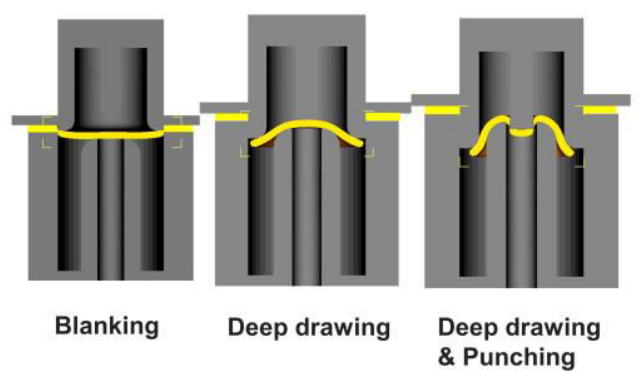

(c)

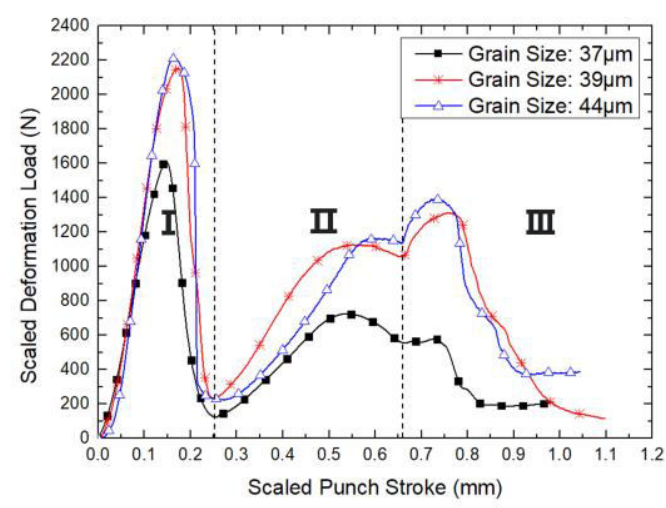

(b)

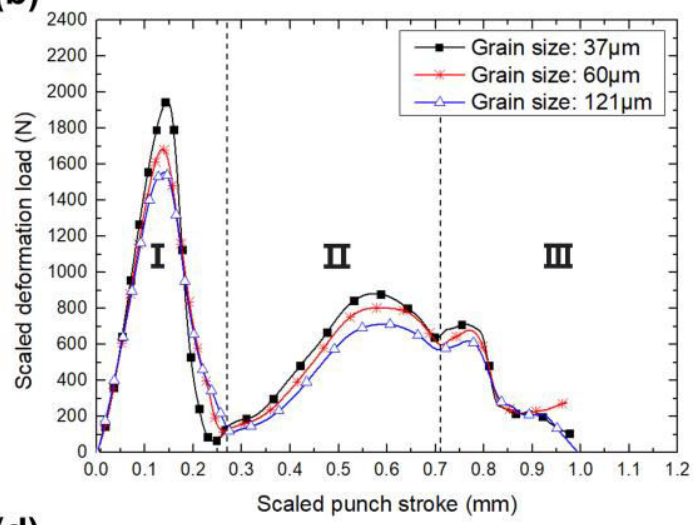

(d)

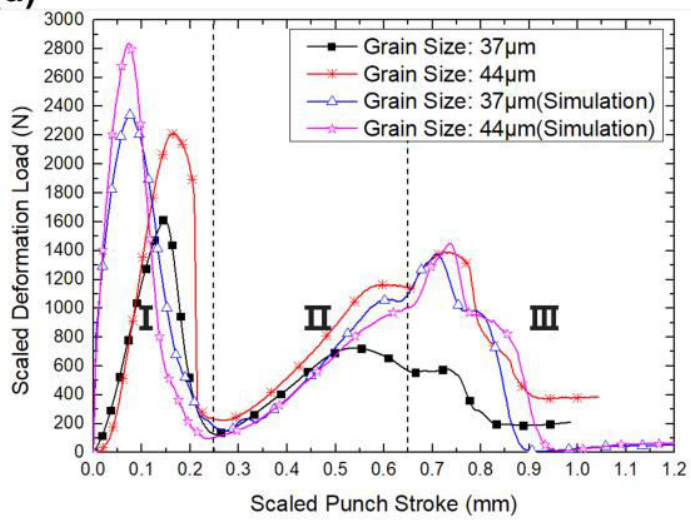

Fig. 5 (a) Illustration of the three operations in the process. Load-stroke curves in different conditions: (b) Results with the same thickness and various annealing 
conditions. (c) Results with various thicknesses and the same annealing condition. (d) Results of physical experiments and FE simulations

\subsection{Dimensional accuracy}

Dimensional accuracy is one of the most critical factors in considering the quality of the deformed parts. There are many factors affecting dimensional accuracy, such as grain inhomogeneity, complex friction conditions, etc. The variation of thicknesses of the formed cups was measured, through the marked reference points on the cross-section, as shown in Fig. 6 (a). In order to obtain accurate experimental results, the thicknesses of the same marked points on both sides were averaged, and the results were obtained by averaging multiple sets of data. The data were also normalized as shown in Eq. (3).

$$
\text { Scaled Thickness }=\frac{\text { Thickness } * 0.5}{\alpha}
$$

Fig. 6 (b-d) show the thicknesses of various grain sizes and thicknesses. 
(a)
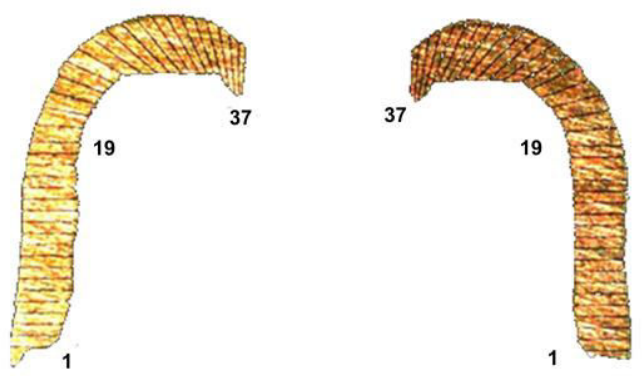

(c)

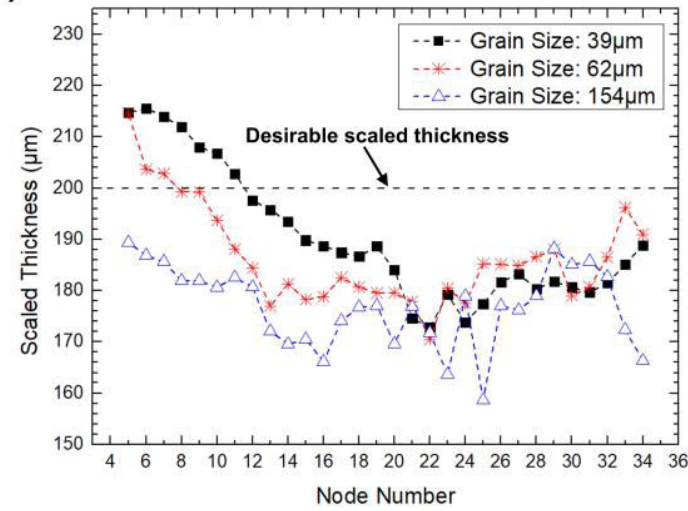

(b)

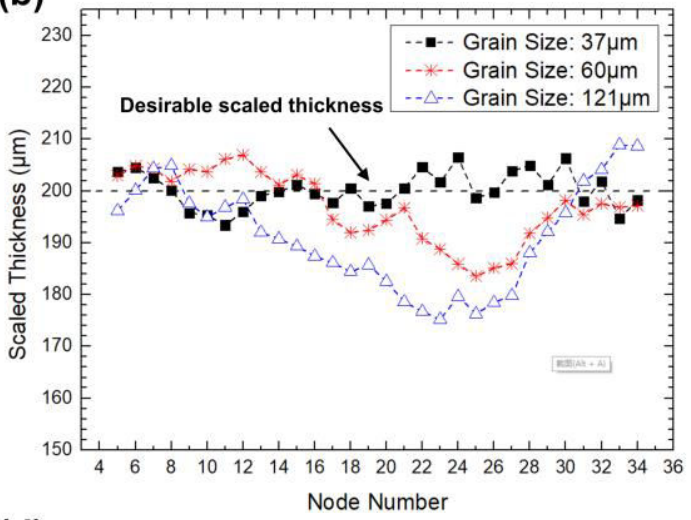

(d)

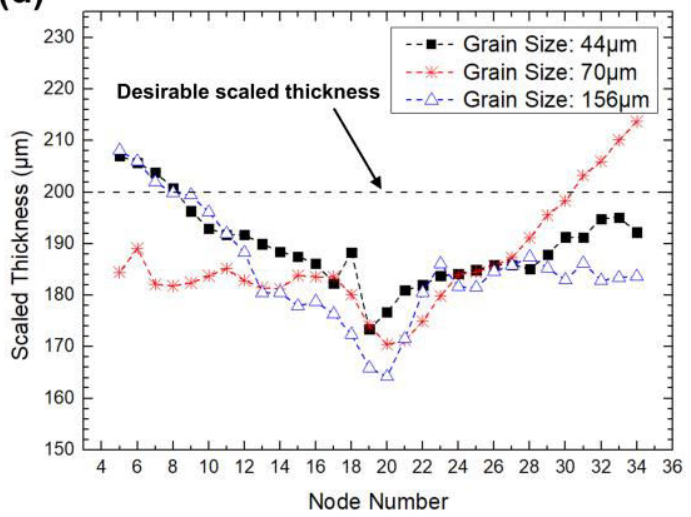

Fig. 6 (a) Schematic diagram of the cross-sectional marking points of the part. The scaled thicknesses of the cups at various marker points with the thickness of (b) 0.2

$$
\mathrm{mm}, \text { (c) } 0.4 \mathrm{~mm} \text {, and (d) } 0.6 \mathrm{~mm}
$$

The trends of thickness variation are similar for different thicknesses. There is the valley of the data at around point 22 . With the increase of grain size, the variations in thickness fluctuation become greater. Therefore, Eq. (4) is established for calculating the scaled thickness variation for the further study of the fluctuation, where $x_{n}$ is the discrepancy between the scaled thickness and the desirable scaled thickness.

$$
\text { Scaled thickness variation }=\frac{\sum_{i=1}^{n}\left|x_{n}\right|}{n}
$$

Fig. 7 shows the scaled thickness variation for various thicknesses and grain sizes. Their trends are similar for different thicknesses. The variation in thickness is induced 
by the inhomogeneous properties of grains, which means that the flow stress of each grain is not identical in the same direction of the force. Thickness variation is increased with grain size, which can be explained by grain boundary strengthening theory. For the sheets with the same thickness, the larger grain size promotes uneven deformation, since there are fewer grain boundaries in the sheet with larger grains, so there are fewer constraints on the grain. However, the variations between various thicknesses are irregular. For meso-scaled parts, the variation in thickness is decreased as the thickness of the sheet is increased because the surface grains are less constrained. Parts made from $0.6 \mathrm{~mm}$ sheets have a more significant proportion of internal grains with similar grain sizes. Therefore, the parts are more resistant to deformation and have a relatively smaller variation in thickness. The thickness variation of $0.2 \mathrm{~mm}$ sheets is much smaller than those of the other two groups. This phenomenon may be related to complex friction conditions with part size scaling down due to the OLP and CLP theories and the differences in the deformation load in the second and third stages leading to the undesirable material deformation.

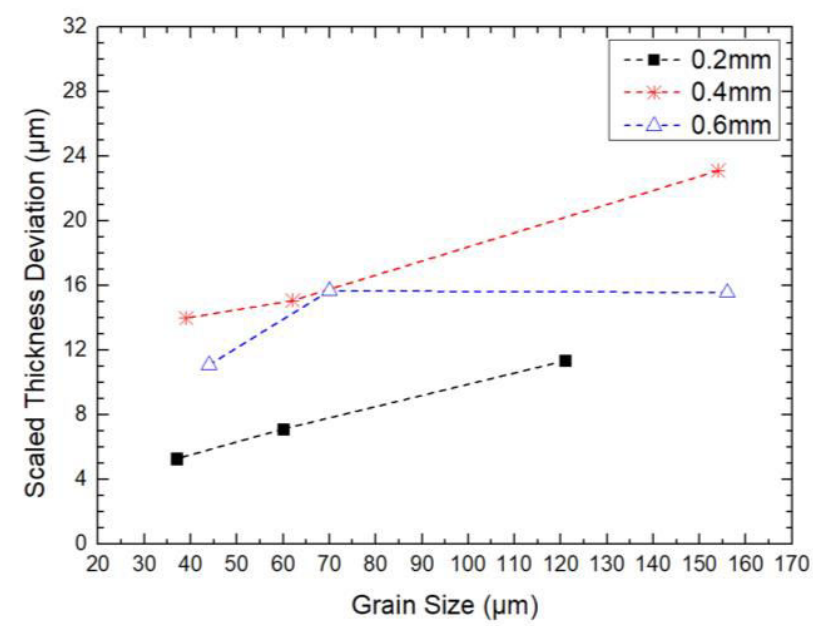

Fig. 7 The scaled thickness variations of cups with various thicknesses and grain sizes

\subsection{Influence of processes}

It is critical to investigate the flow behaviors of materials in deep drawing process, which determines the dimensional accuracy of the deformed parts and further affects whether the part can be assembled and used properly. The results in Fig. 6 
show that the parts become thinner in the bending area. To find the causes of this phenomenon, the flow behaviors of materials were investigated through experiments and simulations, as shown in Fig. 8. The flows of the materials at the shape are divided into two opposite directions, which are all tangential to the thickness direction, leading to the thinning area. Besides, the materials in other areas nearly flow along the thickness direction, resulting in less variation in thickness. To compare the thickness variations in the thinning area with different grain sizes and thicknesses, the thickness variations were calculated for the data at points 18-25, as shown in Fig. 9. Although the trends are the same as the overall thickness variations, the thickness variation of the sheets with the thickness of $0.2 \mathrm{~mm}$ is dramatically larger for the materials with larger grains. For the sheets of the thickness of $0.2 \mathrm{~mm}$, there are only 2-3 grains along the thickness direction. According to grain boundary strengthening theory and surface layer model, the grain boundaries are less dense and there is a greater proportion of surface grains, the constraint to the deformation of grains is less. When the direction of material flow is tangential to the thickness direction, it is easier to make the parts thinner.
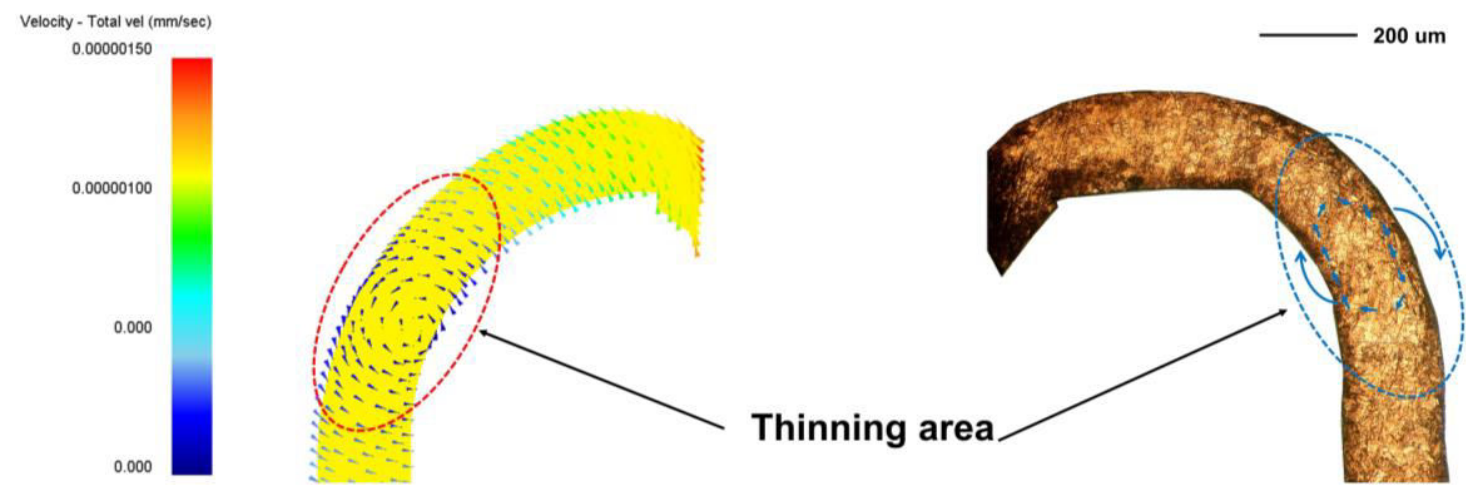

Fig. 8 The flow behavior of the material annealed at $500^{\circ} \mathrm{C}$ for 1 hour with the thickness of $0.2 \mathrm{~mm}$ 


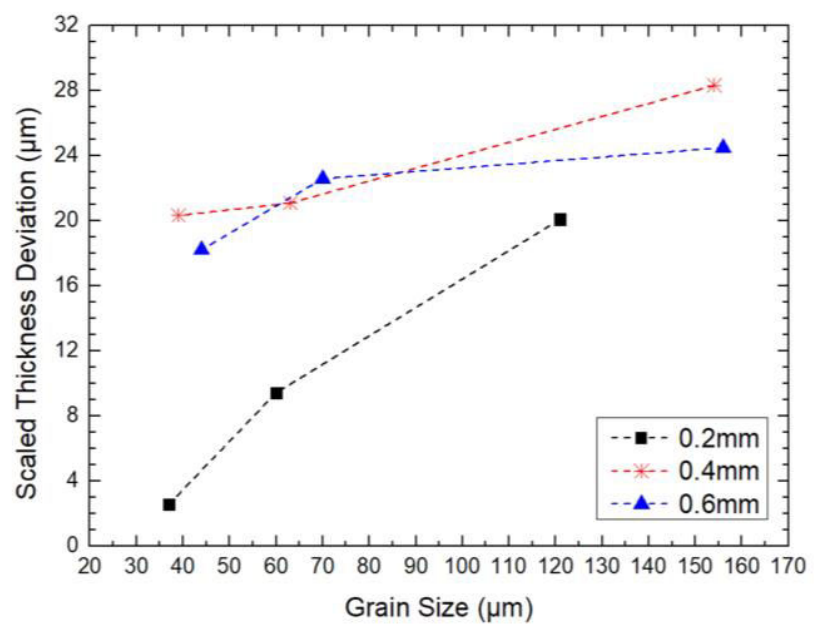

Fig. 9 The scaled thickness variations of the cups with various thicknesses and grain sizes in the thinning area

Secondly, it is an essential objective of this study to investigate whether the punching operation affects the forming of the cups. The compound forming system which did not have the upper punch was used to produce the cup without a hole, and other conditions were the same. The same method for measuring the thickness of the cup without a hole was adopted to observe the influence of punching operation. A comparison of the thicknesses of the two types of parts made from $0.2 \mathrm{~mm}$ sheets is shown in Fig. 10. The trend in thickness variation is essentially the same for both types, and the difference in values is not significant. Furthermore, thickness variations are observed as shown in Fig. 11. The thickness variations of the two parts are also similar. Thus, the punching operation does not significantly affect the thicknesses of the parts. 


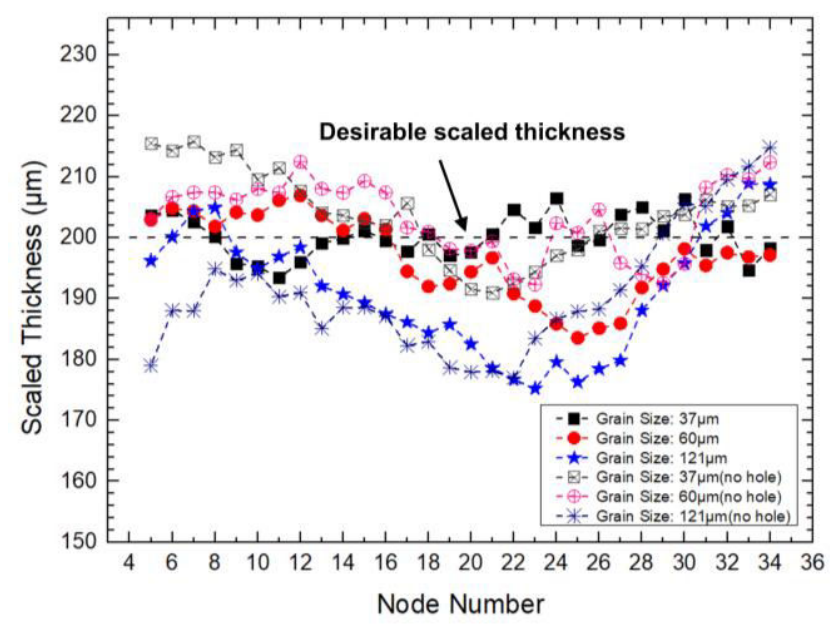

Fig. 10 Comparison of the thickness of the micro-scaled central-punched cups and the cups without the hole made from the sheets with different grain sizes

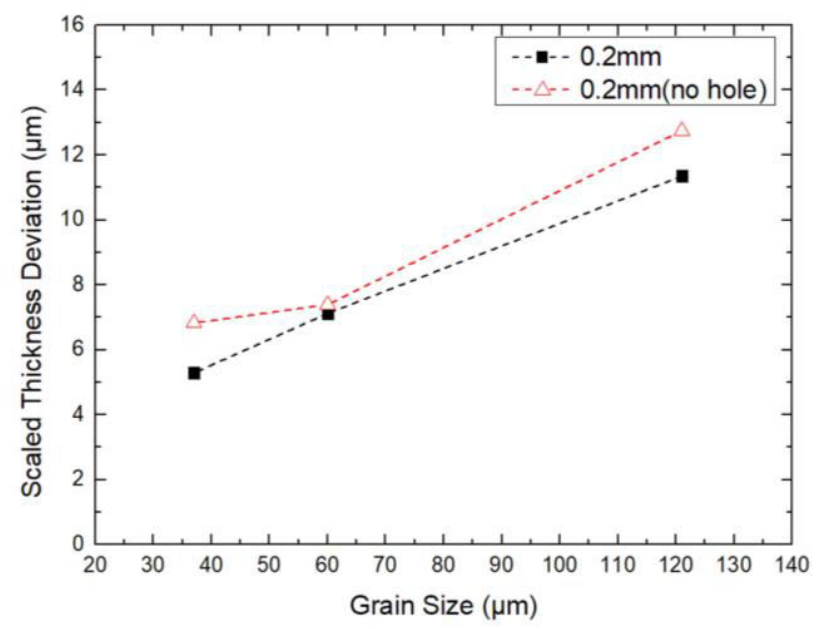

Fig. 11 Comparison of the thickness variations of the micro-scaled central-punched cups and the cups without the hole made from the sheets with different grain sizes

The hole diameter was also investigated to confirm if the geometrical and grain sizes affect the hole dimension. The parts with the size factor of 0.5 and 1.5 were examined, and the diameters shown in Fig. 12 (a) were calculated following Eq. (5).

$$
\text { Scaled Diameter }=\frac{\text { Diameter } * 0.5}{\alpha}
$$

Fig. 12 (b) shows that grain sizes and size scales barely influence the diameter of the hole, which may be caused by the clearance between the insert die and upper punch. 
As shown in Fig. 8, the materials flow is almost opposite to the direction of movement of the upper punch, which prevents the expanding deformation. Therefore, the punching operation does not significantly affect the diameter of the hole of the parts. Thus, a certain amount of clearance can guarantee the accuracy of the hole forming.
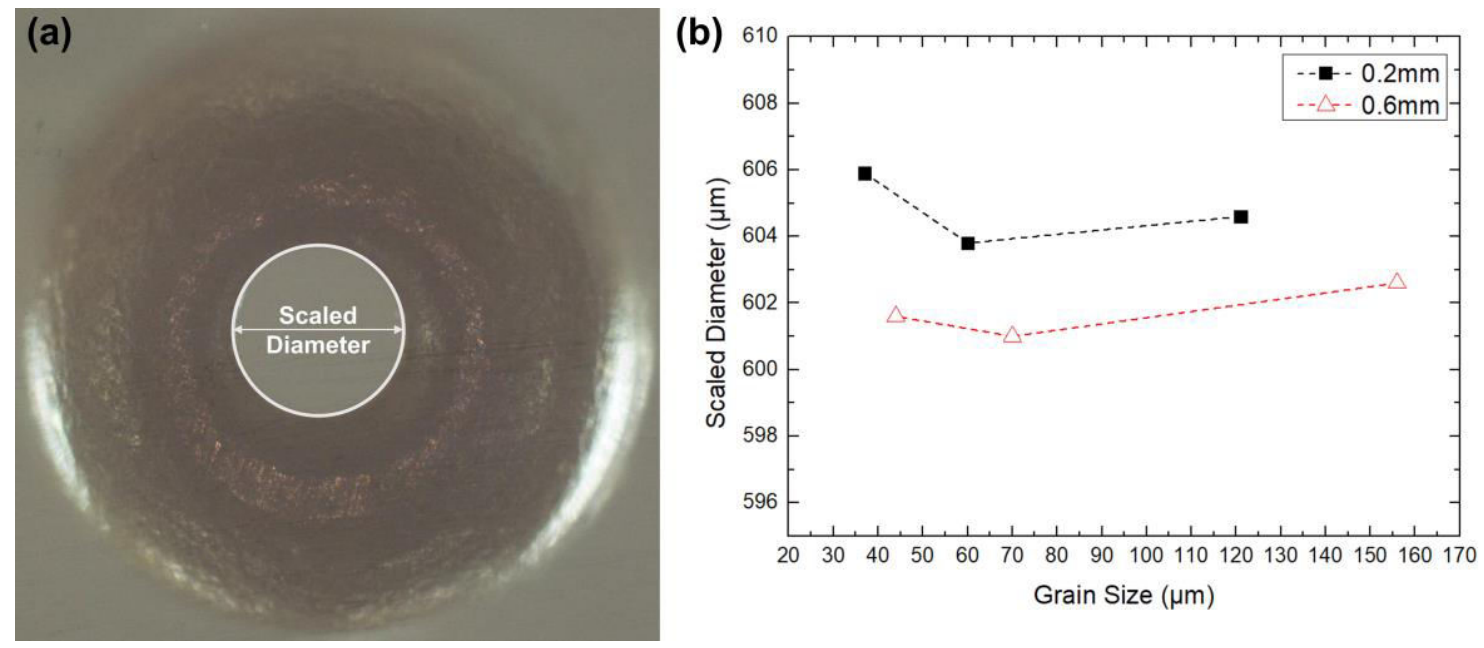

Fig. 12 Comparison of the diameters of the hole fabricated by punching with the size factor of 0.5 and 1.5

\subsection{Surface defects}

The wrinkles on the surface are caused by deformation instability under the compressive stress and they are a primary surface defect in deep drawing process [36]. The defects identified by SEM on the inner and outer walls of the formed cups with various thicknesses and grain sizes are shown in Fig. 13. The micro-scaled cups with fine grains have the best surface quality in this research, which may have similar reasons for the small thickness variations of the micro-scaled components. For the meso-scaled cups with different thicknesses and similar grain sizes, wrinkles are more obvious on the thinner cups. Wrinkles are mainly caused by a single variable which is the inhomogeneous deformation of grains in this research [37]. For the thinner cups, their constraint on surface grains is less, and they have fewer internal grains, leading to the formation of visible wrinkles. However, wrinkles on the cups with the same 
thickness become more significant with the sheets with larger grain size induced by the inhomogeneity of properties of larger grains.

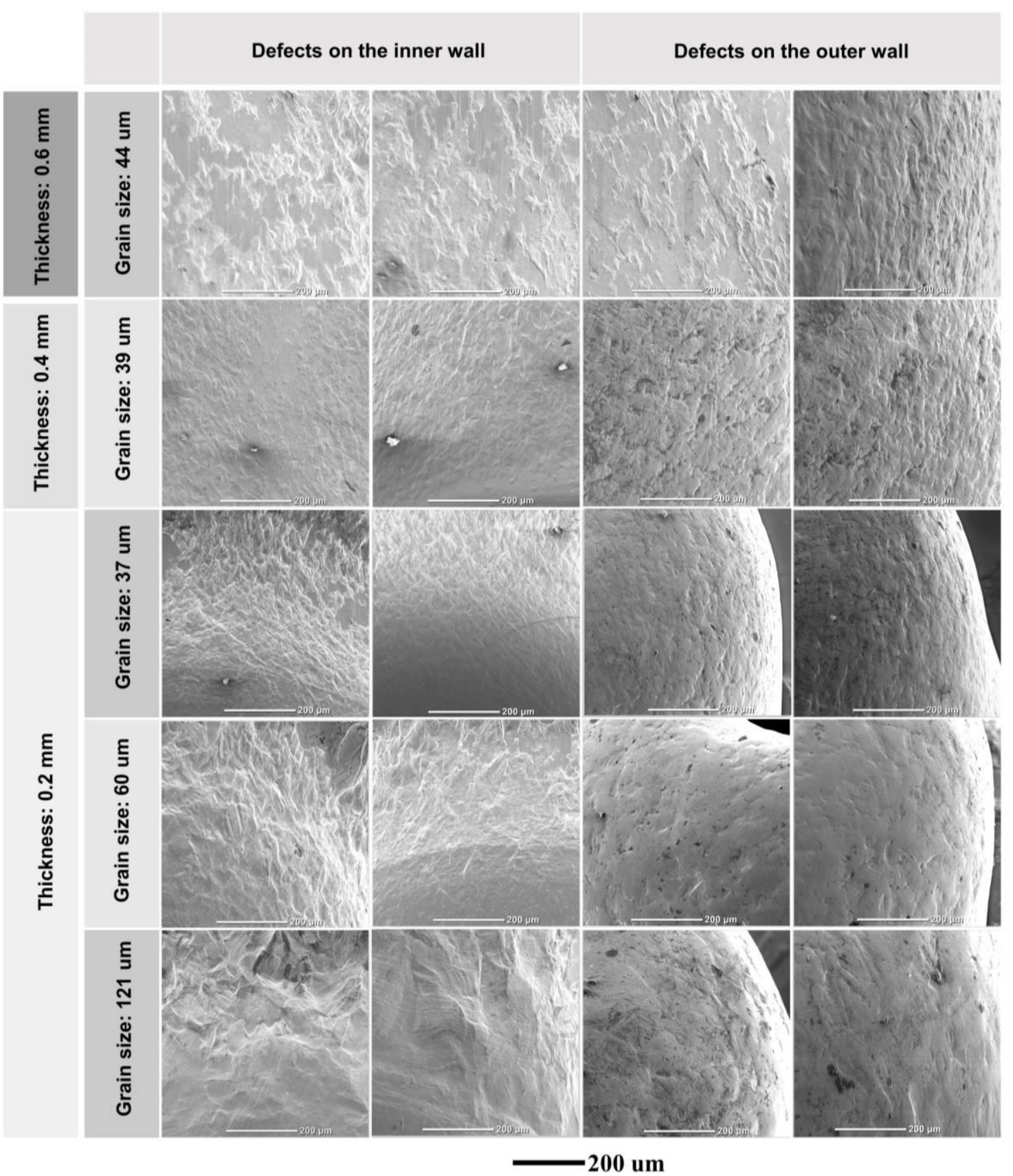

Fig. 13 SEM pictures of the surface quality of central-punched cups with various thicknesses and grain sizes

\section{Conclusions}

In this research, a compound forming system combining deep drawing, punching and blanking for producing central-punched cups in microscale and mesoscales using 
the sheets having various grain sizes was designed and developed. The deformation behaviors and dimensional accuracy of the formed parts with different thicknesses and grain sizes were investigated through physical experiments and FE simulations. The results of the physical experiment and FE simulation were compared. The following conclusions can then be drawn.

(1) The compound forming system can fabricate central-punched cups within one stroke. Its feasibility was verified and this process is reliable for making multi-scaled central-punched cups.

(2) For the cups with similar grain size, the forming load is increased with the thickness. The deformation load is reduced with the increase of grain size under the same thickness condition. Moreover, the ultimate forming load of micro-scaled cups in the drawing-punching stage is smaller than that in the single-drawing stage. The phenomenon of meso-scaled parts is the opposite. The load-stroke results of simulations are similar to the experimental ones, though there are deviations.

(3) In terms of dimensional accuracy, the variation of the part thickness is within a desirable range thickness and there is a thinning area. For the sheet with larger grain size scenario, the thickness variation of the deformed cups becomes larger. However, the parts with different thicknesses are not in consistent with the expected variation, which means that the variation is increased with the decrease of thickness, where the thickness variation of micro-scaled parts is much smaller than those of meso-scaled parts. With the increase of thickness for meso-scaled cups, their scaled thickness variation is decreased with the similar grain size. In addition, the variation in the thinning areas is more significant for microscale.

(4) The thickness of the cup without a hole is approximately the same as that of the central-punched cups, and their thickness variations are approximately similar. Besides, grain and part sizes barely influence the diameter of the hole due to the prevention of tooling clearance. Thus, hole punching has little effect on the dimensional accuracy of the central-punched cups.

(5) The main type of defects on the surface of the central-punched cups is the 
wrinkle. In this research, the surface quality of micro-scaled parts is better than that of meso-scaled ones, and the wrinkles become severe with the decrease of thickness for meso-scaled cups. Furthermore, the size of wrinkles is correlated to grain size. It is found in this research that the micro-scaled cups with fine grains have the best surface quality.

\section{Acknowledgments}

The authors would like to acknowledge the funding support to this research from the NSFC key project of No. 51835011 of the National Natural Science Foundation of China, the project of ZE1W from The Hong Kong Polytechnic University, and the project of 15223520 from the General Research Fund of Hong Kong Government.

\section{Competing Interests}

The authors declare that there are no known competing financial interests or personal relationships that would have appeared to influence the work reported in this research.

\section{Author Contributes}

Dien HU: Design, Data collection and analysis, First draft, review and editing. Jun-yuan Zheng: Conceptualization, Validation, Draft review. M.W. Fu: Conceptualization, Supervision, Draft review.

\section{References}

[1] J. Cao, E. Brinksmeier, M.W. Fu, R.X. Gao, B. Liang, M. Merklein, M. Schmidt, J. Yanagimoto (2019) Manufacturing of advanced smart tooling for metal forming. CIRP Annals 68: 605-628.

[2] A.R. Razali, Y. Qin (2013) A review on micro-manufacturing, micro-forming and their key issues. Procedia Engineering 53: 665-672.

[3] C.P. Raja, T. Ramesh (2021) Influence of size effects and its key issues during microforming and its associated processes-a review. Engineering Science and Technology, an International Journal 24: 556-570.

[4] M.W. Fu, W.L. Chan (2013) A review on the state-of-the-art microforming technologies. The International Journal of Advanced Manufacturing Technology 67: 2411-2437.

[5] M.W. Fu, M. Yong, K. Tong, T. Muramatsu (2006) A methodology for evaluation of metal forming system design and performance via CAE simulation. International journal of production research 44: 1075-1092. 
[6] X. Lai, L. Peng, P. Hu, S. Lan, J. Ni (2008) Material behavior modelling in micro/meso-scale forming process with considering size/scale effects. Computational Materials Science 43: 1003-1009.

[7] L. Peng, X. Lai, H.-J. Lee, J.-H. Song, J. Ni (2009) Analysis of micro/mesoscale sheet forming process with uniform size dependent material constitutive model. Materials science and engineering: A 526: 93-99.

[8] G. Wang, J. Han, Y. Lin, W. Zheng (2021) Investigation on size effect of surface roughness and establishment of prediction model in micro-forming process. Materials Today Communications 27: 102279.

[9] H. Zhang, J. Liu, D. Sui, Z. Cui, M.W. Fu (2018) Study of microstructural grain and geometric size effects on plastic heterogeneities at grain-level by using crystal plasticity modeling with high-fidelity representative microstructures. International Journal of Plasticity 100: 69-89.

[10] M. Klein, A. Hadrboletz, B. Weiss, G. Khatibi (2001) The 'size effect'on the stress-strain, fatigue and fracture properties of thin metallic foils. Materials Science and Engineering: A 319: 924-928.

[11] H. Zhang, X. Dong (2016) Experimental and numerical studies of coupling size effects on material behaviors of polycrystalline metallic foils in microscale plastic deformation. Materials Science and Engineering: A 658: 450-462.

[12] W.L. Chan, M.W. Fu (2012) Experimental and simulation based study on microscaled sheet metal deformation behavior in microembossing process. Materials Science and Engineering: A 556: 60-67.

[13] Z. Jiang, J. Zhao, H. Lu, D. Wei, K.-i. Manabe, X. Zhao, X. Zhang, D. Wu (2017) Influences of temperature and grain size on the material deformability in microforming process. International Journal of Material Forming 10: 753-764.

[14] G. Feng, D. Sagapuram (2021) A strong basis for friction as the origin of size effect in cutting of metals. International Journal of Machine Tools and Manufacture 103741.

[15] W.L. Chan, M.W. Fu, J. Lu (2008) An integrated FEM and ANN methodology for metal-formed product design. Engineering Applications of Artificial Intelligence 21: 1170-1181.

[16] L. Luo, Z. Jiang, D. Wei, F. Jia (2021) A study of influence of hydraulic pressure on micro-hydromechanical deep drawing considering size effects and surface roughness. Wear 203803.

[17] A. Molotnikov, R. Lapovok, C. Gu, C.H.J. Davies, Y. Estrin (2012) Size effects in micro cup drawing. Materials Science and Engineering: A 550: 312-319.

[18] W.L. Chan, M.W. Fu, J. Lu, L.C. Chan (2009) Simulation-enabled study of folding defect formation and avoidance in axisymmetrical flanged components. Journal of Materials Processing Technology 209: 5077-5086.

[19] W.L. Chan, M. Fu (2013) Meso-scaled progressive forming of bulk cylindrical and flanged parts using sheet metal. Materials \& Design 43: 249-257.

[20] J. Xu, B. Guo, C. Wang, D. Shan (2012) Blanking clearance and grain size effects on micro deformation behavior and fracture in micro-blanking of brass foil. International journal of machine tools and manufacture 60: 27-34. 
[21] J.-Y. Zheng, H. Yang, M.W. Fu, C. Ng (2019) Study on size effect affected progressive microforming of conical flanged parts directly using sheet metals. Journal of Materials Processing Technology 272: 72-86.

[22] J.-Y. Zheng, S. Shi, M.W. Fu (2020) Progressive microforming of pin-shaped plunger parts and the grain size effect on its forming quality. Materials \& Design 187: 108386.

[23] J.-Y. Zheng, J. Wang, M.W. Fu (2021) Experimental and numerical study of the size effect on compound Meso/Microforming behaviors and performances for making bulk parts by directly using sheet metals. Journal of Manufacturing Processes 66: 506520.

[24] D. Raabe, Y. Wang, F. Roters (2005) Crystal plasticity simulation study on the influence of texture on earing in steel. Computational Materials Science 34: 221-234.

[25] W.T. Li, M.W. Fu, J.L. Wang, B. Meng (2016) Grain size effect on multi-stage micro deep drawing of micro cup with domed bottom. International Journal of Precision Engineering and Manufacturing 17: 765-773.

[26] L. Luo, D. Wei, G. Zu, Z. Jiang (2021) Influence of blank holder-die gap on micro-deep drawing of SUS304 cups. International Journal of Mechanical Sciences 191: 106065 .

[27] C.-H. Chen, J.-T. Gau, R.-S. Lee (2009) An experimental and analytical study on the limit drawing ratio of stainless steel 304 foils for microsheet forming. Materials and Manufacturing Processes 24: 1256-1265.

[28] F. Gong, B. Guo, C. Wang, D. Shan (2011) Micro deep drawing of micro cups by using DLC film coated blank holders and dies. Diamond and Related Materials 20: 196-200.

[29] M.W. Fu, B. Yang, W.L. Chan (2013) Experimental and simulation studies of micro blanking and deep drawing compound process using copper sheet. Journal of Materials Processing Technology 213: 101-110.

[30] F. Vollertsen, Z. Hu (2010) Analysis of punch velocity dependent process window in micro deep drawing. Production Engineering 4: 553-559.

[31] B.-Y. Joo, S.-H. Rhim, S.-I. Oh (2005) Micro-hole fabrication by mechanical punching process. Journal of Materials Processing Technology 170: 593-601.

[32] Z. Gao, L. Peng, P. Yi, X. Lai (2015) Grain and geometry size effects on plastic deformation in roll-to-plate micro/meso-imprinting process. Journal of Materials Processing Technology 219: 28-41.

[33] J.R. Greer, J.T.M. De Hosson (2011) Plasticity in small-sized metallic systems: Intrinsic versus extrinsic size effect. Progress in Materials Science 56: 654-724.

[34] Z. Xu, L. Peng, E. Bao (2018) Size effect affected springback in micro/meso scale bending process: Experiments and numerical modeling. Journal of Materials Processing Technology 252: 407-420.

[35] H. Kamali, H. Xie, F. Jia, H. Wu, H. Zhao, H. Zhang, N. Li, Z. Jiang (2019) Effects of nano-particle lubrication on micro deep drawing of $\mathrm{Mg}$-Li alloy. The International Journal of Advanced Manufacturing Technology 104: 4409-4419. 
[36] M.A. Shafaat, M. Abbasi, M. Ketabchi (2011) Investigation into wall wrinkling in deep drawing process of conical cups. Journal of Materials Processing Technology 211: 1783-1795.

[37] J. Zhao, T. Wang, F. Jia, Z. Li, C. Zhou, Q. Huang, Z. Jiang (2021) Experimental Investigation on Micro Deep Drawing of Stainless Steel Foils with Different Microstructural Characteristics. Chinese Journal of Mechanical Engineering 34: 1-11. 